\title{
Morphology and formation mechanism of metallic inclusions in VB-grown sapphire crystals
}

\author{
T. Taishi ${ }^{1}$, T. Kobayashi ${ }^{2}$, M. Shinozuka ${ }^{2}$, E. Ohba ${ }^{1,2}$, C. Miyagawa ${ }^{1,2}$, K. Hoshikawa $^{1}$
}

${ }^{1}$ Faculty of Engineering, Shinshu University, Wakasato, Nagano 380-8533, Japan

${ }^{2}$ Fujikoshi Machinery Corp., 1650 Kiyono Matsushiro-machi, Nagano 381-1233, Japan

Morphologies of metallic inclusions observed in sapphire crystals grown by the vertical Bridgman (VB) technique using a tungsten (W) crucible were investigated. Square- or hexagonal-shaped inclusions $2-5 \mu \mathrm{m}$ in size were observed in sapphire crystals around the interface between the seed and the grown crystal. It was found that such inclusions consisted of W metal used for the crucible. The morphology of some of the inclusions reflects a rhombic dodecahedron which is based on the cubic structure of $\mathrm{W}$ and is surrounded by $\{110\}$ faces. It is probable that inclusions form in the sapphire melt during the crystal growth process, and then sink in the melt to the growth interface due to the high density of $\mathrm{W}$. 
Keywords: A1: Defect, A1: Inclusion, A2: Bridgman technique, A2: Growth from melt, B1: Sapphire, B3: Light emitting diodes 


\section{Introduction}

In recent years, $c$-plane 0001 sapphire substrates [1] have been used commercially for the fabrication of GaN-based LED devices by the metal-organic chemical vapor deposition (MOCVD) process on account of their relatively good lattice match. Major growth techniques for growing large-size practical sapphire crystals are the Kyropoulos (KP) method [1, 2], heat exchange method (HEM) [1-3], edge-defined, film-fed growth (EFG) $[1,2,4]$, and Czochralski (CZ) method [1, 2, 5]. Recently, we succeeded in the growth of $c$-axis sapphire crystals by the vertical Bridgman (VB) technique using a molybdenum (Mo) or a tungsten (W) crucible [6].

With regard to microscale defects in sapphire crystals, small bubbles $2-10 \mu \mathrm{m}$ in size in EFG-sapphire crystals [7,8] and in CZ-sapphire crystals [9] have been reported. However, there have been few reports with regard to solid inclusions in sapphire crystals. Rectangular and hexagonal inclusions $1-5 \mu \mathrm{m}$ in size in EFG-sapphire crystals have been reported [1]. Danko et al. also reported hexagonal-shaped microparticles $1-5 \mu \mathrm{m}$ in size in sapphire crystals grown by the horizontal directed crystallization method in $\mathrm{CO}$ or $\mathrm{Ar}+\mathrm{CO}$ ambient using graphite hot zones [10]. In both reports, the inclusion was concluded to be $\mathrm{AlAl}_{2} \mathrm{O}_{4}$ which has a spinel structure. However, no experimental data about the composition of the inclusions were reported. 
Microscale defects such as bubbles or a high density of inclusions would influence the yield and efficiency of LED devices, so research into such defects and the reduction of defect density is very important.

This paper reports inclusions observed in sapphire crystals grown by the VB technique using a $\mathrm{W}$ crucible and graphite hot zones in an $\mathrm{Ar}$ ambient. The morphologies and the composition of inclusions were investigated. As results, we found metallic inclusions originating from the crucible, which are of a similar size but of a quite different composition to those previously reported $[1,10]$. Based on experimental results, a possible mechanism for the formation of inclusions is discussed.

\section{Experimental procedure}

Sapphire crystals, $50 \mathrm{~mm}$ in diameter and elongated along the $c$-axis, were grown by the VB technique using a tapered seed and a W crucible [11]. The furnace consisted of a graphite heater and carbon heat shields, and was filled with an atmosphere of $99.999 \%$ pure argon gas (absolute pressure about $100 \mathrm{kPa}$ ). The crucible was slowly drawn downwards at $3 \mathrm{~mm} / \mathrm{h}$, the crystal growth being controlled by the rate at which the crucible was drawn down. Detailed growth conditions have been reported elsewhere [11]. 
One of these boules was cut into wafers $1.5 \mathrm{~mm}$ in thickness. The wafers were lapped and polished on both sides in order to remove any damage due to cutting. The polished sapphire substrates were evaluated using green laser scattering at a wavelength of $532 \mathrm{~nm}$. After confirmation of the distribution of defects detected by this light-scattering, inclusions in the sapphire wafers were examined using an optical microscope or a laser scanning microscope. The detailed morphology was observed using a field emission scanning electron microscope (FE-SEM), and the composition of the inclusions was evaluated using energy dispersive X-ray spectrometry (EDS) working in the FE-SEM.

\section{Results and discussion}

Figure 1 (a) shows a sapphire crystal, $50 \mathrm{~mm}$ in diameter, grown by the VB technique using a tapered seed. A wafer cut vertically from the crystal was used for observation. A light-scattered image of the wafer is shown in Fig. 1 (b), and microscale defects were observed around the interface between the seed and the grown crystal in the area indicated by a white circle. Figure 1 (c) shows a typical optical microscope image of two inclusions found in the white circle shown in Fig 1 (b). A hexagonal- and a square-shaped inclusion were observed as shown in Fig. 1 (c). Figures 2 (a) - (c) show 
typical laser scanning microscope images of inclusions in sapphire crystals grown using a W crucible. Two-dimensional shapes of inclusions observed were square, hexagon and a wide hexagon incorporating a bright lozenge, as shown in Figs. 2 (a), (b) and (c), respectively. These results imply that the morphology of some inclusions was a rhombic dodecahedron as shown in Fig. 2 (d). The inclusions observed were 2 - $5 \mu \mathrm{m}$ in size.

Figure 3 (a) shows a back-scattered electron image of an inclusion in the sapphire wafer shown in Fig. 1 (b). The lighter color of the hexagonal inclusion, compared with the sapphire matrix, indicates that the composition of the inclusion is considerably higher than that in the matrix. Figure 3 (b) shows intensities of EDS analysis in the image shown in Fig. 3 (a). There are three peaks corresponding to Al- $\mathrm{K}_{\alpha}, \mathrm{O}-\mathrm{K}_{\alpha}$ and $\mathrm{W}-\mathrm{M}_{\alpha}$, indicating that the inclusion and surrounding portion of the matrix consisted of $\mathrm{Al}, \mathrm{O}$ and $\mathrm{W}$ atoms. Figures 3 (c), (d) and (e) show EDS mapping images of $\mathrm{Al}, \mathrm{O}$ and $\mathrm{W}$, respectively, corresponding to the inclusion shown in Fig. 3 (a). In the inclusion the intensities of $\mathrm{Al}$ and $\mathrm{O}$ atoms were weak, while that of $\mathrm{W}$ atoms was very strong. Therefore, we conclude that such inclusions consist of $\mathrm{W}$ atoms.

The crystal structure of $\mathrm{W}$ is a body-centered cubic lattice. Considering such a structure and the experimental results obtained, it is likely that the morphology of some inclusions reflects the $\{110\}$ rhombic dodecahedron which represents the form of the 
cubic body centered structure showing, on its surfaces, the highest atomic density.

Such inclusions could be detected only around the interface between a seed and the grown crystal, and no inclusions were found in the main body of the sapphire crystal. Based on these experimental results, one of the possible formation models is as follows. First, molecules including $\mathrm{W}$ enter the sapphire melt coming from the crucible. The contamination process of the $\mathrm{W}$ molecules in the melt is not clear, but the following internal and external routes are proposed as likely mechanisms. In the internal case, free $\mathrm{W}$ atoms are produced by a decomposition reaction induced by residual impurities in the raw materials. In the external case, gas molecules including $\mathrm{W}$, such as $\mathrm{WO}_{3}$, contaminate the melt from the atmosphere in the furnace, and these then resolve into $\mathrm{W}$ atoms in the melt. Further investigation of such a mechanism is now in progress. Next, $\mathrm{W}$ atoms in the melt aggregate and precipitate, due to supersaturation of $\mathrm{W}$ atoms, as $\mathrm{W}$ metal with the cubic structure. The two dimensional hexagonal or square shapes observed in Fig. 1(c) and Fig. 2 reveal that such precipitates were not formed during the cooling process but were formed during the crystal growth in the melt, because they have different orientations. Inclusions featuring the $<100\rangle$-directions probably developed in the sapphire melt during the growth at a temperature higher than the melting point of sapphire $(2323 \mathrm{~K})$, resulting in the shape of a rhombic dodecahedron 
surrounded by $\{110\}$ faces, which corresponds to the lozenge plane shown in Fig. 2 (a). Finally, the $\mathrm{W}$ inclusions so formed sink in the melt to the interface between the seed and the grown crystal due to the greater density of $\mathrm{W}\left(\rho_{\mathrm{W}}=19.3 \mathrm{~g} / \mathrm{cm}^{3}\right)$ [12] compared with that of sapphire melt $\left(\rho_{\text {melt }}=3.0 \mathrm{~g} / \mathrm{cm}^{3}\right)[13]$.

When compared with the inclusions of $\mathrm{AlAl}_{2} \mathrm{O}_{4}$ previously reported by Akselrod [1] and Danko et al. [10], the shape and the size we observed are similar, but the composition of the inclusions is quite different. In such previous reports, information of detailed growth conditions such as the material of the crucible was not clearly shown. The proportion of oxygen in $\mathrm{AlAl}_{2} \mathrm{O}_{4}$ is slightly smaller than that in an $\mathrm{Al}_{2} \mathrm{O}_{3}$ matrix. This indicates that $\mathrm{AlAl}_{2} \mathrm{O}_{4}$ inclusions were probably generated from oxygen vacancies by the reduction of $\mathrm{Al}_{2} \mathrm{O}_{3}$, especially using $\mathrm{CO}$ gas in the growth [10]. In the present growth technique using a graphite heater and heat shields, $\mathrm{CO}$ gas might be generated unintentionally by the existence of residual oxygen in the furnace. However, further comparison with the $\mathrm{W}$ metal inclusions obtained in the present study is impossible because detailed growth conditions in the previous reports $[1,10]$ are unknown.

In our current study, similar inclusions were also observed in VB-sapphire crystals grown using a Mo crucible. Details of the mechanism for the formation of the inclusions in sapphire crystals with and quantitative studies is now in progress and will be reported 
elsewhere.

\section{Conclusions}

Inclusions in VB-grown sapphire crystals in a W crucible have been investigated.

Square or hexagonal-shaped inclusions $2-5 \mu \mathrm{m}$ in size were observed in sapphire crystals but only around the interface between the seed and the grown crystal. Such inclusions consisted of $\mathrm{W}$ metal used as the crucible. The morphology of some of inclusions can be related to that of the $\{110\}$ rhombic dodecahedron which is, not by chance, the form showing the highest surface density in the cubic centered lattice. According to experimental results, a possible mechanism for the formation of inclusions is proposed. It is likely that inclusions form in the sapphire melt during the crystal growth, and then sink in the melt to the interface between the seed and the grown crystal due to higher density of $\mathrm{W}$. These investigations would be useful for further detailed analysis of the formation mechanism and methods for the suppression of inclusions in sapphire crystals. 


\section{References}

[1] M.S. Akselrod, F.J.Bruni, Modern trends in crystal growth and new applications of sapphire, Journal of Crystal Growth 360 (2012) 134-145.

[2] V.A. Tatartchenko, Sapphire crystal growth and applications, in:P.Capper (Ed.), Bulk Crystal Growth of Electronic, Optical and Optoelectronic Materials, John Wiley\&Sons Ltd.,England, 2005, pp.299-338 (Chapter10).

[3] C.P. Khattak,F. Schmid, Growth of the world's largest sapphire crystals, Journal of Crystal Growth 225 (2001) 572-579.

[4] H.E. La Belle Jr., EFG, the invention and application to sapphire growth, Journal of Crystal Growth 50 (1980) 8-17.

[5] A.E. Paladino, B.D. Roiter, Czochralski growth of sapphire, Journal of the American Ceramic Society 47 (1964) 465.

[6] C. Miyagawa, T. Kobayashi, T. Taishi, K. Hoshikawa, Demonstration of crack-free c-axis sapphire crystal growth using the vertical Bridgman method, Journal of Crystal Growth 372 (2013) 95-99.

[7] K. Wada, K. Hoshikawa, Growth and characterization of shaped sapphire crystals, Journal of Crystal Growth $\mathbf{5 0}$ (1980) 151-159.

[8] I. Nicoara, O. M. Buroiu, D. Vizman, Voids engulfment in shaped sapphire crystals, Journal of Crystal Growth 287 (2006) 291-295. 
[9] H. Li, E.A. Ghezal, A. Nehari, G. Alombert-Goget, A. Brenier, K. Lebbou, Bubbles defects distribution in sapphire bulk crystals grown by Czochralski technique, Optical Materials 35 (2013) 1071-1076.

[10] A. Ya. Danko, N. S. Sidelnikova, G. T. Adonkin, A. T. Budnikov, S. V. Nizhankovskii, S. I. Krivongov, The mechanism of light scattering centers' formation in sapphire crystals grown in gas atmosphere, Functional Mater. 10 (2003) 217-223.

[11] K. Hoshikawa, T. Taishi, E. Ohba, C. Miyagawa, T. Kobayashi, J. Yanagisawa, M. Shinozuka, Vertical Bridgman growth of sapphire -Seed crystal and seeding processes-, Proceedings ICCGE-17, Warsaw, Poland, 2013, Journal of Crystal Growth.

[12] K. S. Mohammed, A. Rahmat, A. Aziz, Journal of Materials Processing Technology 213 (2013) 1088-1094.

[13] C. H. Chen, J. C. Chen, C. W. Lu, C. M. Liu, Numerical simulation of heat and fluid flows for sapphire single crystal growth by the Kyropoulos method, Journal of Crystal Growth 318 (2011) 162-167. 


\section{Figure caption}

Fig. 1 (a) Photograph of a VB-grown sapphire crystal, 2 inches in diameter, grown using a tapered W crucible. (b) Light-scattered image of a sapphire wafer cut vertically from the crystal shown in Fig. 1 (a). (c) Optical microscopic image of two inclusions present in the white circle shown in Fig. 1 (b). The shapes of the inclusions are a hexagon on the left and a square on the right.

Fig. 2 Laser scanning microscopic images of (a) square, (b) hexagonal, and (c) hexagonal with lozenge inclusions in a VB-sapphire crystal grown using a W crucible. (d) Illustration of a rhombic dodecahedron as a possible morphology of such inclusions.

Fig. 3. (a) Back-scattered image of an inclusion in a sapphire crystal grown using a W crucible. (b) Intensities of EDS analysis for the image shown in Fig. 3 (a). Corresponding EDS mapping images of related elements of (c) Al, (d) O and (e) W, for the region around the inclusion. 

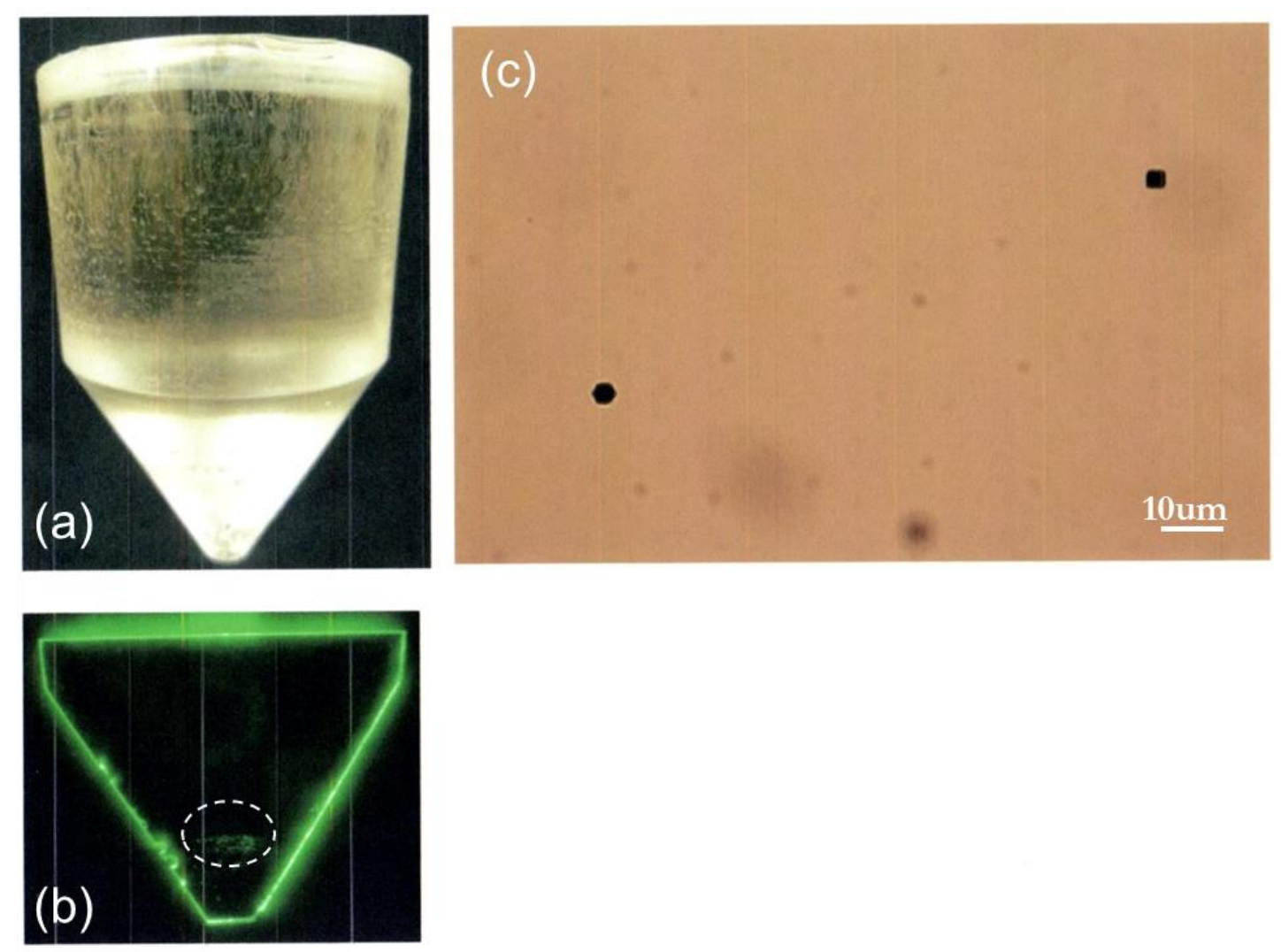

Fig. 1 


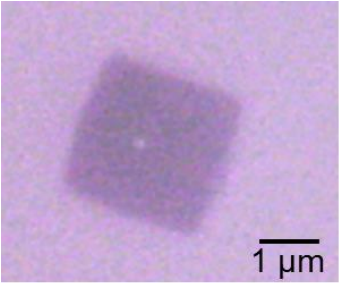

(a)

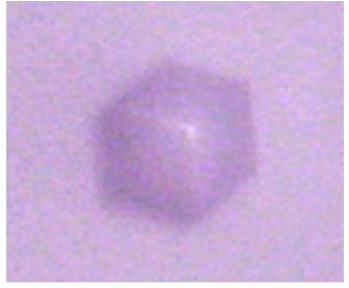

(b)

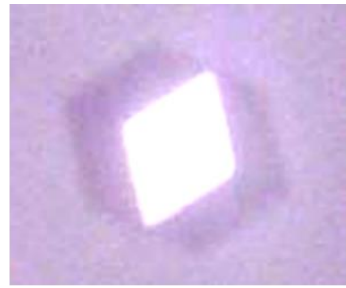

(c)



(d)

Fig. 2 

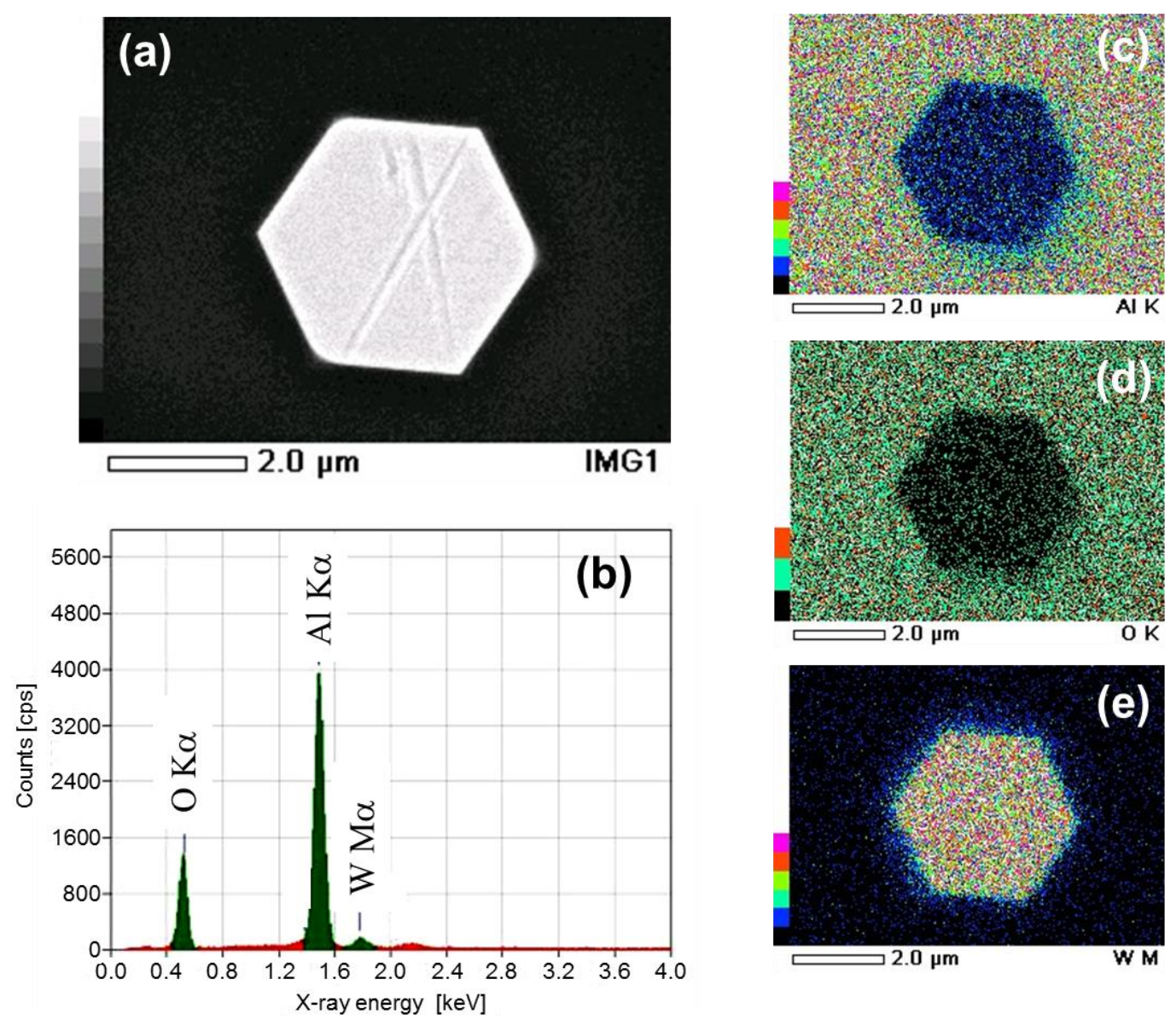

Fig. 3 